\title{
Gutes Schulmanagement steigert den Bildungserfolg
}

\section{David Bader}

\section{Relevanz}

Auch im Bildungswesen braucht es ein leistungsfähiges Management, um die Produktivität zu steigern und aus dem Steuergeld mehr herauszuholen. Es gilt, das richtige Personal auszuwählen, klare Ziele vorzugeben, Leistungsanreize zu setzen, die Zielerfüllung zu kontrollieren, und die Abläufe zu korrigieren, wenn die Leistung nicht stimmt. Die Schulleitung muss autonom handeln und an allen Hebeln drehen können, wenn sie Wirkung erzielen und Leistung verantworten soll. Ein leistungsfähiges Management macht es möglich, bei gleichem Einsatz von Steuergeld den Bildungserfolg zu steigern.

Christian Keuschnigg und Michael Kogler

\section{Quelle}

Der nachfolgende Text ist eine Zusammenfassung von: Bloom, Nicholas, Renata Lemos, Raffaella Sadun und John Van Reenen (2015), Does Management Matter In Schools, Economic Journal 125, 647-674.

Die Leistungen der Schüler fallen unterschiedlich aus. Der Bildungserfolg unterscheidet sich zwischen einzelnen Schulen und verschiedenen Ländern. Nicht nur die pädagogische Qualität der Lehrpersonen und die Grösse und Ausstattung der Klassen bestimmen den Bildungserfolg. Schulen brauchen auch ein gutes

D. Bader $(\bowtie)$

Universität St. Gallen, St. Gallen, Schweiz

E-Mail: david.bader@student.unisg.ch

(C) Der/die Autor(en) 2018

C. Keuschnigg (Hrsg.), Inklusives Wachstum und wirtschaftliche Sicherheit, https://doi.org/10.1007/978-3-658-21344-2_8 
Management. Kann ein leistungsfähiges Schulmanagement den Bildungserfolg steigern? Die Wirtschaftswissenschaft konnte signifikante Zusammenhänge zwischen der Qualität des Managements und der Leistungsfähigkeit von Universitäten in Forschung und Lehre nachweisen. Über die Managementqualität in den Schulen gibt es jedoch nur wenige verlässliche Daten. Es ist daher noch ungewiss, welchen Einfluss das Management auf den Bildungserfolg haben könnte.

Ein Team von Wissenschaftlern um Nicholas Bloom und John Van Reenen ging dieser Frage nach. Sie entwickelten ein international vergleichbares Mass für die Managementqualität von Schulen und analysierten deren Auswirkungen auf den Bildungserfolg der Schülerinnen und Schüler. Sie untersuchten rund 1800 zufällig ausgewählte Schulen in acht Ländern: Deutschland, Grossbritannien, Italien, Schweden, USA, Kanada, Indien und Brasilien. Mithilfe strukturierter Interviews ermittelten sie, inwieweit in den Schulen tatsächlich die besten Managementpraktiken zum Einsatz kommen. Dabei unterschieden sie über 20 verschiedene Dimensionen des Managements wie Formulierung von Zielen, Kontrolle der Zielerreichung, Personalführung und Personalentwicklung oder Planung und Organisation. Die Antworten wurden in eine fünfteilige Skala umgewandelt, welche die Managementqualität misst. Schliesslich verglichen sie die Managementdaten an verschiedenen Schulen mit den jeweiligen Prüfungsergebnissen aus standardisierten Leistungstests.

Moderne Managementmethoden sind an Schulen wenig verbreitet. Die durchschnittliche Managementqualität erreicht nur 2,27 von 5 Punkten.

Eine erste Auswertung der Daten zeigt, dass moderne Managementmethoden in den Schulen bisher nur teilweise Einzug gehalten haben. Anhand der Interviewergebnisse ermittelten die Forscher die durchschnittliche Managementqualität mit 2,27 von maximal fünf Punkten. Nach Abb. 1 waren die Schulen in Grossbritannien führend. Sie erreichten durchschnittlich 2,9 Punkte und lagen damit knapp vor den Schulen in Schweden, Kanada und den USA. Die Managementqualität in Deutschland lag im Mittelfeld, während sie in Italien, Brasilien und besonders in Indien niedrig war. Internationale Unterschiede in der Managementqualität sind im Bildungssektor deutlich stärker ausgeprägt als in anderen Wirtschaftszweigen. Vergleicht man verschiedene Schulen innerhalb eines Landes, so ergibt sich z. B. für Indien ein aussergewöhnlich ungünstiges Bild. Lediglich $3 \%$ der Schulen in den westlichen Ländern, aber $82 \%$ der indischen Schulen verfügten über ein schlechtes Management mit weniger als zwei Punkten auf der fünfteiligen Qualitätsskala. Verglichen mit anderen Branchen sind diese Werte besonders schlecht. 


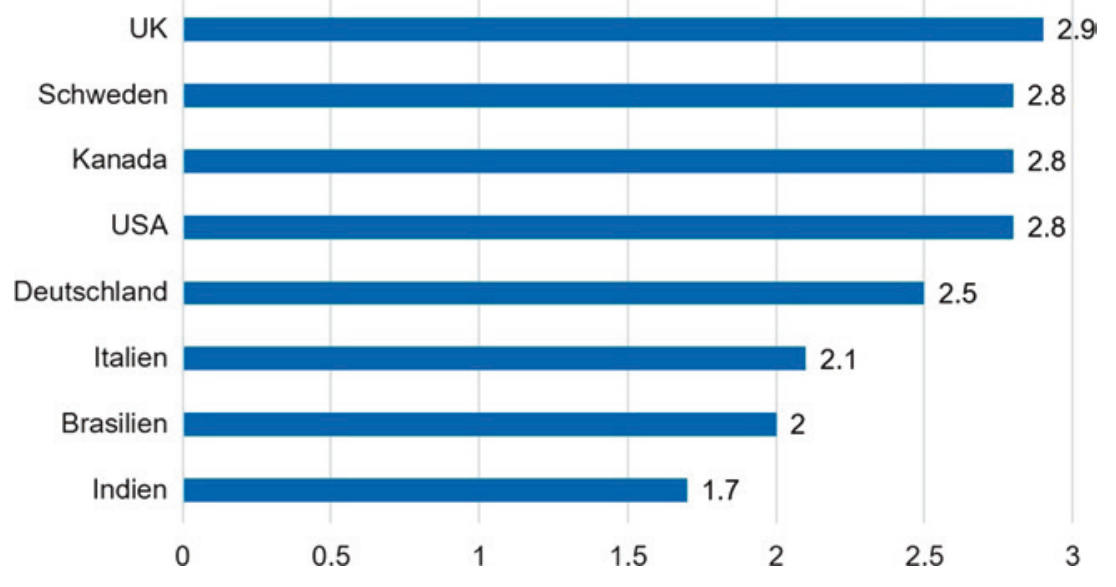

Abb. 1 Durchschnittliche Qualität des Schulmanagements auf einer Skala von 1 (am schlechtesten) bis 5 (am besten). (Quelle: Bloom u. a., 2015, 656)

So hatten $22 \%$ der indischen Unternehmen des verarbeitenden Gewerbes und $10 \%$ in der Gesundheitsbranche eine Managementqualität von mehr als drei Punkten auf der Qualitätsskala. Aber keine der untersuchten Ausbildungsstätten in Indien erzielte mehr als drei Punkte für die Managementqualität.

Warum ist die Managementqualität an den Schulen so niedrig? Eine mögliche Erklärung sind die grossen Defizite im Personalmanagement, angefangen von den Entscheidungen über die Anstellung bzw. Entlassung von Lehrkräften bis zur Bezahlung und zur Beförderungspolitik. In allen untersuchten Ländern erreichte die Bewertung des Personalmanagements lediglich 0,2 bis 0,6 Punkte auf der fünfteiligen Qualitätsskala. Führungsentscheide in anderen Bereichen wie z. B. Planung, Zielsetzung und Kontrolle bewerteten die Forscher hingegen besser.

Wie wirken sich solche Unterschiede in der Managementqualität auf die Leistungen der Schülerinnen und Schüler aus? Der Bildungserfolg einer Schule kann anhand der durchschnittlichen Prüfungsergebnisse von standardisierten Leistungstests gemessen werden. Die empirischen Resultate zeigen einen positiven Zusammenhang zwischen den schulischen Leistungen und der Qualität des Schulmanagements. Steigt die Managementqualität einer Schule um eine Standardabweichung (vergleichbar mit dem Unterschied zwischen Italien und den USA), dann verbessern sich die schulischen Leistungen um gut $42 \%$ einer Standardabweichung. Berücksichtigt man weitere Einflussfaktoren wie z. B. 
den Schultyp, beträgt der Leistungsanstieg immer noch rund $23 \%$ einer Standardabweichung. Der Effekt des Managements ist knapp halb so gross wie jener, der mit einer gezielten Auswahl der Schülerinnen und Schüler aufgrund ihrer vergangenen Leistungen erreicht wird.

Gutes Schulmanagement kann die Leistungen der Schülerinnen und Schüler um 23 bis $42 \%$ einer Standardabweichung verbessern.

Worauf sind die Unterschiede in der Managementqualität von Schulen innerhalb eines Landes zurückzuführen? Die Forscher betonen, dass der Schultyp eine zentrale Rolle spielt. Sie unterscheiden zwischen Privatschulen, regulären staatlichen Schulen und autonomen staatlichen Schulen. Letztere sind Institutionen, welche zumindest teilweise vom Staat finanziert werden, jedoch hinsichtlich Curriculum, Zulassung und Personal über ein erhebliches Mass an Autonomie verfügen. Autonome staatliche Schulen weisen in der Regel die beste Managementqualität auf. Der Unterschied im Vergleich zu den regulären staatlichen Schulen entspricht rund $13 \%$ der Differenz zwischen indischen und britischen Schulen. Die Wissenschaftler führen dies auf verbesserte Prozesse im täglichen Management zurück, wobei den Schuldirektoren eine besondere Rolle zukommt. Die Rechenschaftspflicht gegenüber den Interessengruppen der Schule sowie Führungsstärke in Form der langfristigen Umsetzung einer sorgfältig formulierten Strategie erklären ungefähr die Hälfte des Unterschieds in der Managementqualität relativ zu den anderen zwei Schultypen. Bei autonomen staatlichen Schulen besteht zudem eine negative Korrelation zwischen modernen Managementmethoden und der Anzahl Schüler pro Lehrer. Schulen mit weniger Schüler pro Lehrer verfügen oft über höhere Ressourcen und sind besser in der Lage, fortschrittliche Managementpraktiken durchzusetzen.

Staatliche Schulen verfügen über die beste Managementqualität, wenn sie autonom über Personal, Zulassung und Curriculum entscheiden können.

Die Schulen eines Landes unterscheiden sich also ganz erheblich in der Qualität ihres Managements. Auch zwischen verschiedenen Ländern bestehen grosse Unterschiede. Moderne Managementpraktiken sind im Schulbereich noch wenig verbreitet. Vor allem das Personalmanagement stellte eine häufige Schwäche dar. Die Handlungsempfehlungen an die Politik sind eindeutig. Sie soll dafür Sorge tragen, dass die Managementqualität an den Schulen besser wird. Staatliche 
Schulen sollen weitgehend autonom über ihre Strategie und ihre Prozesse entscheiden können. Mit einer Verbesserung der Managementqualität an den Schulen kann der Staat ein erhebliches Potential erschliessen, um die Leistungen der Schülerinnen und Schüler merklich zu steigern.

Open Access Dieses Kapitel wird unter der Creative Commons Namensnennung 4.0 International Lizenz (http://creativecommons.org/licenses/by/4.0/deed.de) veröffentlicht, welche die Nutzung, Vervielfältigung, Bearbeitung, Verbreitung und Wiedergabe in jeglichem Medium und Format erlaubt, sofern Sie den/die ursprünglichen Autor(en) und die Quelle ordnungsgemäß nennen, einen Link zur Creative Commons Lizenz beifügen und angeben, ob Änderungen vorgenommen wurden.

Die in diesem Kapitel enthaltenen Bilder und sonstiges Drittmaterial unterliegen ebenfalls der genannten Creative Commons Lizenz, sofern sich aus der Abbildungslegende nichts anderes ergibt. Sofern das betreffende Material nicht unter der genannten Creative Commons Lizenz steht und die betreffende Handlung nicht nach gesetzlichen Vorschriften erlaubt ist, ist für die oben aufgeführten Weiterverwendungen des Materials die Einwilligung des jeweiligen Rechteinhabers einzuholen.

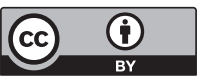

